\title{
Cuffless Blood Pressure Estimation during Exercise Stress Test
}

\author{
S. Mottaghi, M. H. Moradi, and L. Roohisefat
}

\begin{abstract}
Blood Pressure (BP) measurement without using a cuff is an interesting goal especially during Exercise Stress Test. Conventional methods like Cuff-based blood pressure measurement is not accurate enough due to high motion artifacts. High blood pressure during exercise test will cause pain and discomfort during inflation of cuff as well. Many researches have been conducted and shown Pulse Arrival Time (PAT), can be used in blood pressure estimation. This paper proposes a new calibration method for blood pressure estimation during exercise stress test that increases the quality of estimation. Besides PAT and heart rate, estimated blood pressure of previous stage is added as another input parameter. This technique increases the correlation coefficients between real and estimated blood pressures. We also show that by using SPAT, $\triangle H R$ instead of PAT and HR quality of estimation improves.
\end{abstract}

Index Terms-Pulse transit time, pulse arrival time, bruce protocol, exercise stress test, calibration, delta PAT, delta HR.

\section{INTRODUCTION}

Blood Pressure $(B P)$ is a very important vital sign of body that should be measured accurately and periodically for health care purposes. $B P$ measurement contains systolic blood pressure $(S B P)$ and diastolic blood pressure $(D B P)$ measurement. $B P$ can be measured both invasively and non-invasively; $B P$ cannot be measured invasively during exercise stress test. Auscultatory method measures $B P$ by inflation and deflation of a cuff, however this method is time consuming and has pain and also discomfort [1]. Many studies have been conducted on associating biological signals with $B P$ which have focused on Pulse Arrival Time (PAT) [2]-[4].

Exercise stress test is a physical test that challenges and evaluates the performance of the cardiovascular system by putting the body under the physical pressure. Cardiovascular disease is one of the most common illnesses among people. Physicians usually peruse the cardiovascular performance of the patient by exercise stress test before any invasive operation like angiography. Blood pressure monitoring during exercise stress test is very important. Patients might face chest pain or unusual shortness of breath. If any unusual increase, decrease or no change in BP is seen the test should be stopped immediately.

Some researchers have been conducted on relations

Manscript received September 25, 2012; revised October 26, 2012.

S. Mottaghi and M. H. Moradi are with the Department of Biomedical Engieering, Amirkabir University of Technology (Tehran-Polytechnic) (e-mail: soheilmottaghi@aut.ac.ir,mhmoradi@aut.ac.ir).

L. Roohisefat is with the Department of Telecommunication Engieering, K. N. Toosi University of Technology (e-mail: leyla.roohisefat@gmail.com). between $B P$ and PAT during exercise test [5]-[8]. Moreover some models have been introduced as well to estimate $B P$ using PAT [10]-[13]. Self-calibration is proposed in almost all of the methods to tune the outputs [9].

Heart rate also used as an important factor in $B P$ estimation beside the $P A T$. Some studies have empirically observed that for some records in the MIMIC database, $B P$ is highly correlated with instantaneous heart-rate $(H R)$. It has been shown that as heart rate increases, the cardiac output increases as well, and therefore $B P$ increases linearly with $H R$ [9].

\section{MethodolOGY}

This test was conducted on 55 healthy subjects including 24 Females and 31 males, aged between 19 years to 60 years old. No disease has been detected in the subjects in the last 3 months. The subjects were asked not to eat heavy meal and drink beverage for at least 2 hours and 30 minutes before the test. $P P G$ and ECG signals were sampled at $1 \mathrm{KHz}$ and recorded by $A D$ instrument acquisition system (PowerLab /8SP) during the test. Meanwhile $B P$ and HR were measured simultaneously by Bionet Holter (Model: BM1) that the BP measuring module is from SUNTECH company. Exercise stress test has been conducted according to Bruce Protocol test and a treadmill (Model 870A, Ram, Italy) was used for the test. $B P$ was measured on subject's left arm.

Exercise stress test has 6 stages: Rest, Stage 1, Stage 2, Stage 3, 1-minute Recovery and 5-minute Recovery. Speeds and gradients of the treadmill for three stages are listed in Table I. Initially subjects were asked to rest in sitting position for 5 minutes and at the end of this time, recording $E C G$, $P P G$ and $H R$ signals was started. After 20 seconds, $B P$ was measured once. Then subject started walking on treadmill in stage 1. Each stage lasted 3 minutes and at the end of the $3^{\text {rd }}$ minute, subject stood on the border of treadmill for 10 seconds of $P P G$ and ECG noiseless recording. Then 15 seconds $B P$ measurement was carried out. Afterwards, the subject went to stage 2 and this procedure went on. After stage 3 subjects sat and 2 recovery stages as is shown in Fig. 1, were conducted. Fig. 1 shows the protocol of the test has been conducted in this research.

The signals were analyzed offline using Matlab to determine the R-peak of ECG, and foot point of $P P G$. Peak and foot detection algorithm which is introduced in [10] is used in this test. $P A T_{f}$ is the time interval between the R-wave of ECG and the foot point of PPG signal.

We collected 275 datasets in total over 55 subjects. PTT and $H R$ and $B P_{i-1}$ were selected as inputs. $B P$ of previous stages is selected as a set point in order to calibrate the estimation. Before starting the test, blood pressure of subjects is measured once by automatic cuff-based device. 
TABLE I: SPEEDS AND GRADIENTS OF EACH STAGE IN BRUCE PROTOCOL EXERCISE STRESS TEST.

\begin{tabular}{|c|c|c|c|}
\hline Stage & $\begin{array}{c}\text { Speed } \\
(\mathbf{k m} / \mathbf{h r})\end{array}$ & $\begin{array}{c}\text { Speed } \\
(\mathbf{m p h})\end{array}$ & $\begin{array}{c}\text { Gradient } \\
(\boldsymbol{\%})\end{array}$ \\
\hline 1 & 2.74 & 1.7 & 10 \\
\hline 2 & 4.02 & 2.5 & 12 \\
\hline 3 & 5.47 & 3.4 & 14 \\
\hline
\end{tabular}

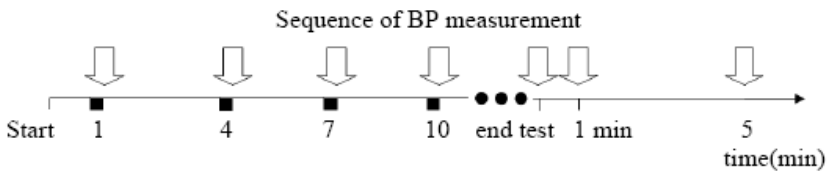

Fig. 1.Time line of the conducted test.

\section{A. Self-Calibration}

Pulse arrival time and heart rate are in relation with blood pressure. By Least Squared estimation method, the inputs-output relation is as:

$$
\begin{gathered}
B P=A \times(P A T)+B \\
B P=A \times(P A T)+B \times H R+C \\
B P=A \times(P A T)+B \times H R+C \times B P_{i-1}+D
\end{gathered}
$$

In this relation, $P A T$ is the averaged of $P A T$ s during 10 seconds standing on the borders in each stage, $H R$ is the heart rate measured by the holter in that time and $B P$ is the blood pressure of previous stage. $B P$ can be first time blood pressure measurement or estimated ones in the next stages. By LS algorithm, coefficients $A, B, C$ and $D$ were calculated. Fig. 2 shows the flow chart of this cuff-less blood pressure measurement.

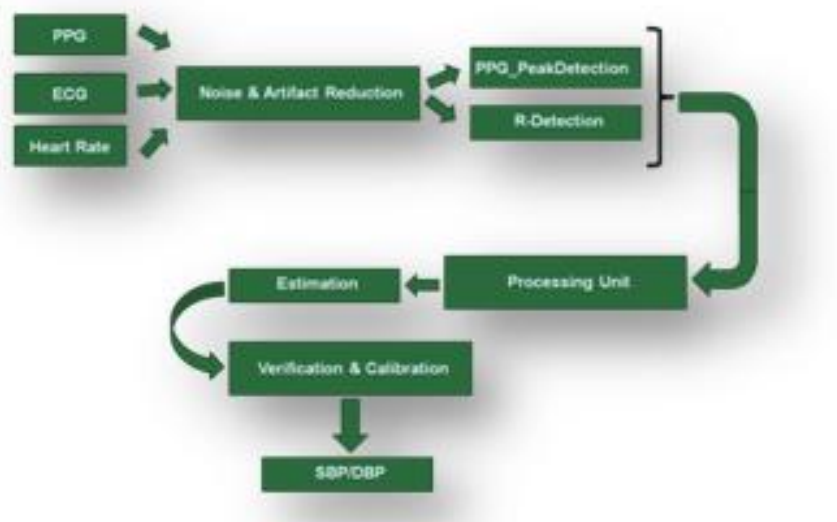

Fig.2. Flowchart of PAT-based blood pressure estimation.

\section{B. Differentiated Inputs with Self-Calibration}

Using $\triangle P A T$ and $\triangle H R$ instead of $P A T$ and $H R$ as inputs, is a method that can be used during exercise stress test. Since the physicians should know the $B P$ every 3 minutes, these differences can be very useful to improve the quality of estimation. LS modeling of blood pressure by using differences are as:

$$
\begin{gathered}
B P=A \times(\Delta P A T)+B \times \Delta H R+C \times B P_{i-1}+D \\
\Delta P T T=P A T_{i}-P A T_{i-1} \\
\Delta H R=H R_{i}-H R_{i-1}
\end{gathered}
$$

Delta means differences between the PAT and $H R$ values of current stage and previous stage. Only one previous stage is considered here.

\section{RESULTS}

Table II compares RMSE of 4 different $L S$ estimators. The first 2 rows are non-calibrated with only PTT and PTT-HR inputs while rows 3 and 4 are calibrated. Fig. 3 shows this comparison better. Calibrated methods have lower errors and delta algorithm has the minimum errors among all algorithms.

TABLE II: RoOT MEAN SQUARED ERROR (RMSE) FOR DIFFERENT ALGORITHMS IN SBP AND DBP.

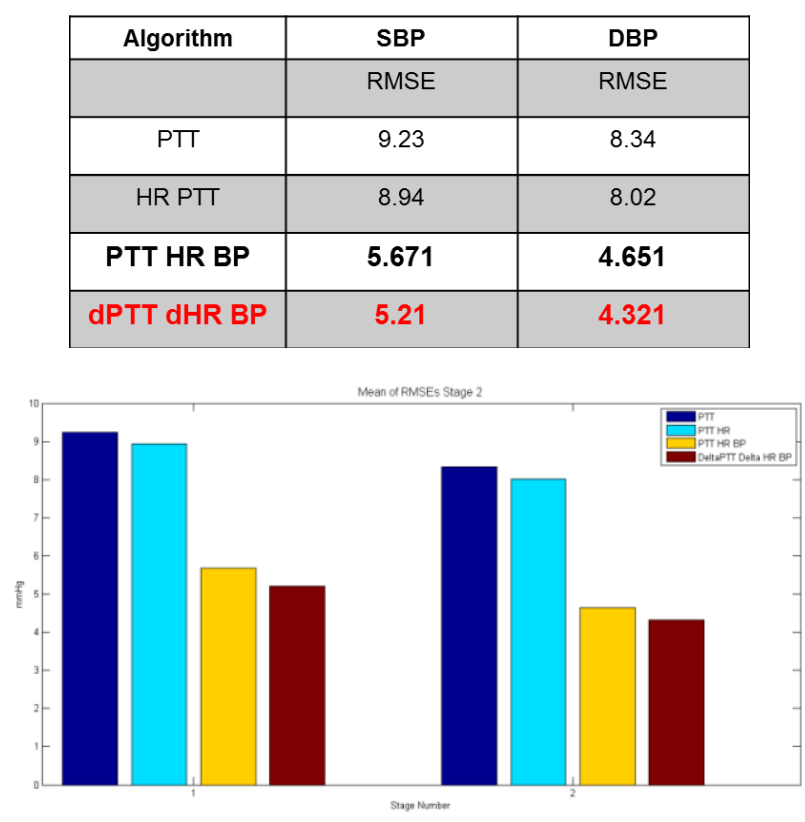

Fig. 3. Bar chart of estimation RMSE for SBP and DBP in Algorithms in Table II.

Table III and Fig. 4 also compare correlation coefficients between real and estimated values of these 4 types of estimators. Correlation coefficients are higher in calibrated algorithm. Delta-calibrated method is the highest.

TABLE III: CORRELATION COEFFICIENT (RMSE) FOR DIFFERENT ALGORITHMS IN SBP AND DBP.

\begin{tabular}{|c|c|c|}
\hline Algorithm & SBP & DBP \\
\hline & Correlation & Correlation \\
\hline PTT & 0.492 & 0.485 \\
\hline HR PTT & 0.522 & 0.499 \\
\hline PTT HR BP & $\mathbf{0 . 6 4 5}$ & $\mathbf{0 . 5 1 2}$ \\
\hline dPTT dHR BP & $\mathbf{0 . 6 9 1}$ & $\mathbf{0 . 5 7 8}$ \\
\hline
\end{tabular}

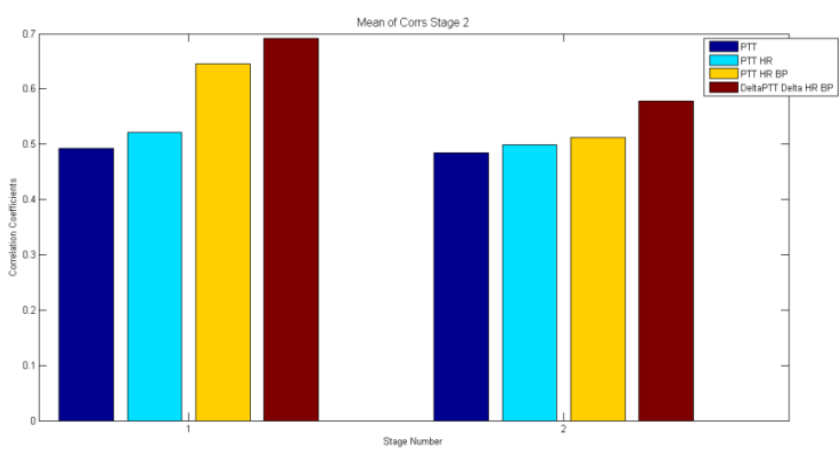

Fig. 4. Bar chart of correlation coefficients. Comparison between real and estimated values for SBP and DBP in algorithms in Table III. 


\section{DisCUSSIONS}

Our results suggest that this calibration method and using $\triangle P A T$ and $\triangle H R$ can improve the quality of blood pressure monitoring during exercise stress test. This method increases the correlation and decreases the errors of both systolic and diastolic blood pressure estimation.

Here are two different comparisons of our estimation algorithms and the result of other researches:

1) Table IV compares correlation coefficients of estimator described by Equation 4 in this article and the result reported in [3]. Researchers have used the recorded signals of 56 subjects in MIMIC database [3]. This result is comparable to rest stages in our project. The RMSE of our non-delta calibrated algorithm is lower compared to the error in [3].

2) In another research conducted in 2007 [9], a fuzzy type II based estimator is used to estimate 10 subjects' Systolic and diastolic blood pressures only using their heart rate. Table $\mathrm{V}$ compares the result of this research with system of Eq9 as the best linear estimation system with the RMSE reported in [9]. Delta-calibrated algorithm has lower errors in both systolic and diastolic blood pressure estimation than reported in [9].

TABLE IV: RoOT MEAN SQUARED ERROR (RMSE) REPORTED IN [3], IN COMPARISON WITH ALGORITHM DESCRIBED IN EQUATION 3 IN SBP AND DBP.

\begin{tabular}{|c|c|c|}
\hline & $\mathbf{S B P}(\mathbf{m m H g})$ & $\mathbf{D B P}(\mathbf{m m H g})$ \\
\hline Algorithm & RMSE & RMSE \\
\hline Ref[3] & 8.36 & 5.9 \\
\hline Eq(3)- Stage1 & 5.24 & 3.71 \\
\hline Eq(3)-StageR1 & 7.1415 & 3.9929 \\
\hline
\end{tabular}

TABLE V: RoOt MEAN SQUARED ERROR (RMSE) REPORTED IN [9], IN COMPARISON WITH ALGORITHM DESCRIBED IN EQUATION 4 IN SBP AND DBP.

\begin{tabular}{|c|c|c|}
\hline & SBP $(\mathbf{m m H g})$ & $\mathrm{DBP}(\mathbf{m m H g})$ \\
\hline Algorithm & $\mathrm{RMSE}$ & $\mathrm{RMSE}$ \\
\hline Ref[1 [9] & 7.032 & 5.2555 \\
\hline Eq(4)- Stage1 & 5.671 & 4.651 \\
\hline Eq(4)-StageR1 & 6.5415 & 3.9929 \\
\hline
\end{tabular}

\section{CONCLUSIONS}

In this paper we introduced a calibration method for cuffless $B P$ estimation using $P A T, H R$ for the exercise stress test application. Cuff-based BP measurement is not accurate during exercise stress test because of high level of motion artifacts and pain caused by inflation of cuff in high blood pressure. Because of simplicity of the algorithm, $B P$ measurement can be very faster and more accurate during physical activities by using this method of calibration. It would be better if the design of the systems becomes age-grouped. Dividing people into age interval groups might increase the accuracy of the estimation. Delta-PAT and delta$H R$ used in this paper has higher correlation with $B P$ than $P A T$ and $H R$ and then can be used in wearable devices for athletes as well. Further studies will be done on design of more intelligent algorithms to develop robust, accurate and reliable devices.

\section{ACKNOWLEDGMENT}

The authors would like to thank Amirkabir University of Technology (Tehran-Polytechnic) for the opportunity to work on this algorithms and device. In particular, the authors would like to express their gratitude to the Faculty of Biomedical Engineering and Arkan Ara Company (Tehran) and Resquake robotics group for their support of this work.

\section{REFERENCES}

[1] Z. Qiao, S. Yang, D. Teng, D. Anh, K. S. Bum, C. Li, J. Basran, V. Dal B. Haas, and C. Younhee, "Pulse transit time-based blood pressure estimation using hilbert-huang transform," in Engineering in Medicine and Biology Society, 2009. EMBC 2009. Annual International Conference of the IEEE, pp. 1785-1788, 2009.

[2] X. F. Teng and Y. T. Zhang, "An evaluation of a PTT-based method for noninvasive and cuffless estimation of arterial blood pressure," in Engineering in Medicine and Biology Society, 2006. EMBS '06. 28th Annual International Conference of the IEEE, pp. 6049-6052, 2006.

[3] F. S. Cattivelli and H. Garudadri, "Noninvasive cuffless estimation of blood pressure from pulse arrival time and heart rate with adaptive calibration," in Wearable and Implantable Body Sensor Networks, 2009. BSN 2009. Sixth International Workshop, pp. 114-119, 2009.

[4] W. B. Gu, C. C. Y. Poon, and Y. T. Zhang, "A novel parameter from PPG dicrotic notch for estimation of systolic blood pressure using pulse transit time," in Medical Devices and Biosensors, 2008. ISSS-MDBS 2008. 5th International Summer School and Symposium, pp. 86-88, 2008.

[5] X. F. Teng, C. C. Y. Poon, and Y. T. Zhang, "Recoverability trend of blood pressure and pulse transit time after treadmill exercise," in Engineering in Medicine and Biology Society, 2005. IEEE-EMBS 2005. 27th Annual International Conference, pp. 3510-3513, 2005.

[6] Y. M. Wong and Y. T. Zhang, "The Effects of Exercises on the Relationship between Pulse Transit Time and Arterial Blood Pressure," in Proceedings of the 2005 IEEE Engineering in Medicine and Biology 27th Annual Conference, Shanghai, China, September 1-4, 2005.

[7] M. Y. Wong, E. P. MacPherson, Y. T. Zhang, and J. C. Cheng, "The effects of pre-ejection period on post-exercise systolic blood pressure estimation using the pulse arrival time technique," Eur J Appl Physiol, vol. 111, pp. 135-44, Jan 2011

[8] Q. Liu, C. C. Y. Poon, M. Y. M. Wong, and Y. T. Zhang, "The time-frequency analysis of variabilities of heart rate, systolic blood pressure and pulse transit time on normotensive subjects after exercise," in Applied Sciences in Biomedical and Communication Technologies, pp. 1-3, 2009.

[9] U. Mahmood, A. A. Jumaily, and M. A. Jaafreh, "Type-2 Fuzzy Classification of Blood Pressure Parameters," in International Co, pp. 595-600, 2007.

[10] J. G. Thomas, "A method for continuously indicating blood pressure," Journal of Physiology, vol. 129, no. 3, pp. 75P-76P, Sept. 1955.

[11] D. A. Newandee, S. S. Reisman, M. N. Bartels, and R. E. D. Meersman, "The effects of respiration on heart rate and blood pressure spectra during exercise below the ventilatory threshold," in Bioengineering Conference, 2002. Proceedings of the IEEE 28th Annual Northeast, pp. 47-48, 2002.

[12] Y. M. Wong and Y. T. Zhang, "The effects of exercises on the relationship between pulse transit time and arterial blood pressure," in Engineering in Medicine and Biology Society, 2005. IEEE-EMBS 2005. 27th Annual International Conference, pp. 5576-5578, 2005.

[13] M. Y. Wong, E. P. MacPherson, and Y. T. Zhang, "The acute effects of running on blood pressure estimation using pulse transit time in normotensive subjects," Eur J Appl Physiol, vol. 107, pp. 169-75, Sep 2009.

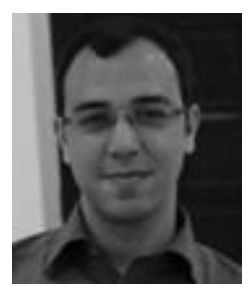

Soheil Mottaghi received the B.Sc. degree in electrical engineering from the K.N.Toosi University of Technology, Tehran, Iran, in 2010 and the M.Sc. degree in biomedical engineering from the Amirkabir University of Technology (Tehran Polytechnic), Tehran, Iran, in 2012. Since 2010, he is working as research assistant in the biomedical instrumentation and signal processing Laboratory. Additionally, he has been distinguished as top researcher in 2009 at K. N. Toosi University of Tech. He has also participated in several industrial projects related to electrical and biomedical 
engineering. His research interests include signal and image processing, rehabilitation and bio-robotics. Mr. Mottaghi has been member of technical committee in some of national and international Robotics competitions since 2010. He has got six different awards in rescue real league in national and international Robocup competitions since 2008.

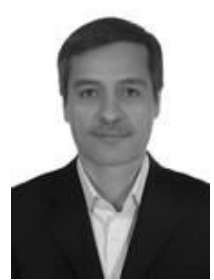

Mohammad Hassan Moradi received the B.S and M.S degrees in electronic engineering from Tehran University, in 1988 and 1990 respectively, and the $\mathrm{PhD}$ degree from the University of Tarbiat Modarres, Tehran, in 1995. He has been with the faculty of biomedical engineering, Amirkabir University of Technology (AUT), since 1995, where he is currently a Professor and Director of Bio-Electric Department. His primary research and teaching interests involve the theory and application of medical instrumentation, biomedical signal processing, wavelet systems design, time-frequency transforms and fuzzy neural systems. He has published over 60 technical papers in international journals, over 200 technical papers in international conferences and is the translator of one book with subject of wavelet signal processing.

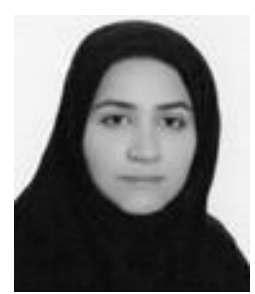

Leyla Roohisefat received the B.Sc. degree in electrical and telecommunication engineering from the K.N.Toosi University of Technology, Tehran, Iran, in 2011. She has worked as a research assistant in Coding Laboratory in Electrical engineering Faculty of K.N.Toosi University of Technology in 2011. Her research interests are digital and biomedical instrumentation and signal processing, coding, cryptography, wireless communications and body networks. She has been in collaboration with the biomedical instrumentation and signal processing Laboratory in 2012 and also working as an electrical engineer in some industrial and research projects since Jan 2011. 